\title{
Origin and Evolution of the Universe
}

\author{
Walter Petry \\ Mathematical Institute of the University Düsseldorf, Düsseldorf, Germany \\ Email:wpetry@meduse.de
}

How to cite this paper: Petry, W. (2021)

Origin and Evolution of the Universe. Journal of Modern Physics, 12, 1749-1757.

https://doi.org/10.4236/jmp.2021.1213102

Received: September 26, 2021

Accepted: November 7, 2021

Published: November 10, 2021

Copyright (c) 2021 by author(s) and Scientific Research Publishing Inc. This work is licensed under the Creative Commons Attribution International License (CC BY 4.0).

http://creativecommons.org/licenses/by/4.0/

\begin{abstract}
A theory of gravitation in flat space-time is shortly summarized and applied to cosmological models. These models start with a gravitational field and without matter. Gravitational energy is converted to matter and the total energy is conserved. The arising universe has no singularity (no big bang) and is not expanding. The redshift is a gravitational effect. It follows by converting gravitational energy to matter changing the gravitational field. This is another gravitation theory different from general relativity which also gives the presently most accepted results of general relativity for weak gravitational fields but has not the problems of general relativity with a big bang.
\end{abstract}

\section{Keywords}

Gravitation, Flat Space-Time, Field Theory, Gravitational Energy, Matter Arises, No Big Bang, Conservation of Total Energy, Non-Expanding Space, Redshift Is a Gravitation Effect

\section{Introduction}

The presently most accepted universe is based on Einstein's general theory of relativity (GR). The application of GR to flat cosmological models gives an expanding universe with a singularity in the beginning, the so-called big bang. The universe must have cosmic inflation in the beginning to explain our big universe.

In this article, a theory of gravitation in flat space-time (GFST) which has till now not received attention by cosmologists is applied to cosmological models. The universe starts with gravitational energy and not with matter. In the course of time, gravitational energy is converted to matter implying the observed redshift of distant objects by virtue of the change of the gravitational field. The total energy is always conserved. The universe is not singular and it doesn't expand. Spherically, symmetric perturbations in the universe can arise in the beginning of the universe and they grow quickly in the matter dominated universe. This 
may explain the big galaxies in the universe whereas the big bang gives only small galaxies in contradiction to observations.

\section{The Universe}

GR is a theory of gravitation giving for weak gravitational fields agreement to experimental accuracy of theory and experiment. This may be the high acceptance of GR by many scientists. GR is also applied to homogeneous, cosmological models to get origin and development of the universe. The universe begins with a big bang (singularity) and it must expand very quickly (cosmic inflation) by virtue of the observed big universe and the many galaxies.

More than forty years ago, in the year 1979, I published a theory of gravitation in flat space-time. This theory can be described by a gravitational field in the pseudo-Euclidean geometry. Gravitation is a field theory and not a geometry as by GR. GFST gives for weak gravitational fields to measurable accuracy the same results as GR. Hence, we get the same acceptance of GFST as GR for weak gravitational fields. But the results of cosmological models are very different by these two theories. The universe starts with uniformly distributed gravitational field by GFST (no big bang). Gravitation is attracting. The densified gravitational field contracts to matter and a part of the gravitational field surrounds the originated object. Every object is surrounded by a gravitational field. Hence, objects attract one another by the surrounded gravitational field and not by their masses. In the course of time matter and other types of energy arise at coasts of gravitational energy. The sum of all types of arising energies is conserved. The universe is not singular and doesn't expand. The redshift follows by converting gravitational field to matter.

\section{Gravitation in Flat Space-Time}

Nearly all cosmologists use GR and the results of this theory to describe and study the universe. This theory is well known and gives a singularity, the big bang in the beginning of the universe. In addition cosmic inflation is needed to explain the observed big universe. Therefore, we will use another theory of gravitation, namely gravitation in flat space-time, to study the universe (compare the book [1]).

This theory is not a geometry as GR but a field theory of gravitation in flat space-time. The metric is given by

$$
(\mathrm{d} s)^{2}=-\eta_{i j} \mathrm{~d} x^{i} \mathrm{~d} x^{j}
$$

where $\left(\eta_{i j}\right)$ is a symmetric tensor. A special case is the pseudo-Euclidean geometry with

$$
\eta_{i j}=\delta_{j}^{i}(i, j=1,2,3), \eta_{i 4}=\eta_{4 i}=0(i=1,2,3), \eta_{44}=-1 .
$$

Here, $\left(x^{i}\right)=\left(x^{1}, x^{2}, x^{3}\right)$ are the Cartesian coordinates and $x^{4} c t$. Put

$$
\eta=\operatorname{det}\left(\eta_{i j}\right) \text {. }
$$


The gravitational field is described by a symmetric tensor $\left(g_{i j}\right)$. Let $\left(g^{i j}\right)$ be defined by

$$
g_{i k} g^{k j}=\delta_{i}^{j}
$$

Put

$$
G=\operatorname{det}\left(g_{i j}\right) .
$$

The proper-time $\tau$ is defined by

$$
(c \mathrm{~d} \tau)^{2}=-g_{i j} \mathrm{~d} x^{i} \mathrm{~d} x^{j} .
$$

The Lagrangean of the gravitational field is given by

$$
L(G)=-\left(\frac{-G}{-\eta}\right)^{1 / 2} g_{i j} g_{k l} g^{m n}\left(g_{/ m}^{i k} g_{/ n}^{j l}-\frac{1}{2} g_{/ m}^{i j} g_{/ n}^{k l}\right) .
$$

Here, the bar/denotes the covariant derivative relative to the flat space-time metric (1).

The Lagrangean of dark energy (given by the cosmological constant $\Lambda$ ) has the form

$$
L(\Lambda)=-8 \Lambda\left(\frac{-G}{-\eta}\right)^{1 / 2}
$$

Let

$$
\kappa=4 \pi k / c^{4}
$$

( $k$ gravitational constant) and assume that matter is a perfect fluid. Then, the mixed energy-momentum tensors of gravitation, of dark energy and of matter for a perfect fluid are

$$
\begin{gathered}
T_{j}^{i}(G)=\frac{1}{8 \kappa}\left[\left(\frac{-G}{-\eta}\right)^{\frac{1}{2}} g_{k l} g_{m n} g^{i v}\left(g_{j}^{k m} g_{v}^{l n}-\frac{1}{2} g_{j}^{k l} g_{v}^{m n}\right)+\frac{1}{2} \delta_{j}^{i} L(G)\right] \\
T_{j}^{i}(\Lambda)=\frac{1}{16 \kappa} \delta_{j}^{i} L(\Lambda) \\
T_{j}^{i}(M)=(\rho+p) g_{j k} u^{k} u^{i}+\delta_{j}^{i} p c^{2} .
\end{gathered}
$$

where $\rho, p$ and $\left(u^{i}\right)$ denote density, pressure and four-velocity of matter. It follows by (6)

$$
c^{2}=g_{i j} u^{i} u^{j}
$$

Let us define the covariant differential operator

$$
D_{j}^{i}=\left[\left(\frac{-G}{-\eta}\right)^{1 / 2} g^{k l} g_{j m} g_{/ l}^{m i}\right]_{/ k} .
$$

Then the field equations for the gravitational potentials $\left(g_{i j}\right)$ have the form

$$
D_{j}^{i}-\frac{1}{2} \delta_{j}^{i} D_{k}^{k}=4 \kappa T_{j}^{i} .
$$

Here, $T_{j}^{i}$ is the sum of the energy-momentum tensors of gravitation, of 
matter and of dark energy

$$
T_{j}^{i}=T_{j}^{i}(G)+T_{j}^{i}(M)+T_{j}^{i}(\Lambda) .
$$

Define the symmetric energy-momentum tensor

$$
T^{i j}(M)=g^{i k} T_{k}^{j}(M),
$$

then the equations of motion are (in covariant form)

$$
T_{i / k}^{k}(M)=\frac{1}{2} g_{k l / i} T^{k l}(M) .
$$

In addition to the field Equation (13) and the equations of motion (16) the conservation of the total energy-momentum holds, i.e.

$$
T_{i / k}^{k}=0 \text {. }
$$

The field Equation (13) are formally similar to those of GR where $D_{j}^{i}$ corresponds to the Ricci tensor and $T_{j}^{i}$ is the total energy-momentum without that of gravitation which is no tensor by GR. These results can be found in the book [1] and in the article [2].

\section{Homogeneous, Isotropic Universe}

We follow along the lines of article [2].

Let us use the pseudo-Euclidean geometry (2), (3) as metric. The matter tensor is given by perfect fluid with

$$
u^{i}=0(i=1,2,3)
$$

and pressure $p$ and density $\rho$ with

$$
p=p_{m}+p_{r}, \rho=\rho_{m}+\rho_{r} .
$$

Here, the indices $m$ and $r$ denote matter and radiation. The equations of state for matter (dust) and radiation are

$$
p_{m}=0, \quad p_{r}=\frac{1}{3} \rho_{r} .
$$

The potentials are given by virtue of (18), homogeneity and isotropy

$$
g_{i j}= \begin{cases}a^{2}(t) & (i=j=1,2,3) \\ -\frac{1}{h(t)} & (i=j=4) \\ 0 & (i \neq j)\end{cases}
$$

The four-velocity is by Equation (18)

$$
\left(u^{i}\right)=(0,0,0, c \sqrt{h}) .
$$

Let $t_{0}=0$ be the present time and assume as initial condition at present

$$
a(0)=h(0)=1, \dot{a}(0)=H_{0}, \dot{h}(0)=\dot{h}_{0}, \quad \rho(0)=\rho_{m 0}, \quad \rho_{r}(0)=\rho_{r 0} .
$$

Here, the dot denotes the time-derivative, $H_{0}$ is the Hubble constant and $\dot{h}_{0}$ is a further constant, $\rho_{m 0}$ and $\rho_{r 0}$ are the present densities of matter and ra- 
diation. It follows by (16) under the assumption that matter and radiation do not interact

$$
\rho_{m}=\rho_{m 0} / \sqrt{h} \rho_{r}=\rho_{r 0} /(a \sqrt{h}) .
$$

The field Equation (13) implies by the use of (21) the two non-linear differential equations

$$
\begin{aligned}
& \frac{\mathrm{d}}{\mathrm{d} t}\left(a^{3} \sqrt{h} \frac{\dot{a}}{a}\right)=2 \kappa c^{4}\left(\frac{1}{2} \rho_{m}+\frac{1}{3} \rho_{r}+\frac{\Lambda}{2 \kappa c^{2}} \frac{a^{3}}{\sqrt{h}}\right), \\
& \frac{\mathrm{d}}{\mathrm{d} t}\left(a^{3} \sqrt{h} \frac{\dot{h}}{h}\right)=4 \kappa c^{4}\left(\frac{1}{2} \rho_{m}+\rho_{r}+\frac{1}{8 \kappa c^{2}} L(G)-\frac{\Lambda}{2 \kappa c^{2}} \frac{a^{3}}{\sqrt{h}}\right)
\end{aligned}
$$

where

$$
L(G)=\frac{1}{c^{2}} a^{3} \sqrt{h}\left(-6\left(\frac{\dot{a}}{a}\right)^{2}+6 \frac{\dot{a}}{a} \frac{\dot{h}}{h}+\frac{1}{2}\left(\frac{\dot{h}}{h}\right)^{2}\right) .
$$

The expression $\frac{1}{16 \kappa} L(G)$ is the density of the gravitational field. The conservation of the total energy gives

$$
\left(\rho_{m}+\rho_{r}\right) c^{2}+\frac{1}{16 \kappa} L(G)+\frac{\Lambda}{2 \kappa} \frac{a^{3}}{\sqrt{h}}=\lambda c^{2}
$$

where $\lambda$ is a constant of integration. The Equations (25), (26) and (27) give by the use of the initial conditions (23)

$$
\frac{\dot{h}}{h}=-6 \frac{\dot{a}}{a}+2 \frac{4 \kappa c^{4} \lambda t+\varphi_{0}}{2 \kappa c^{4} \lambda t^{2}+\varphi_{0}+1}
$$

with

$$
\varphi_{0}=3 H_{0}\left(1+\frac{1}{6} \frac{\dot{h_{0}}}{h_{0}}\right)
$$

Integration of (28) yields

$$
a^{3} \sqrt{h}=2 \kappa c^{4} \lambda t^{2}+\varphi_{0} t+1 .
$$

Equation (27) gives at present time $t_{0}=0$ by using the initial conditions (23)

$$
\frac{1}{3}\left(8 \kappa c^{4} \lambda-\varphi_{0}^{2}\right)=4\left(\frac{8}{3} \pi k\left(\rho_{m 0}+\rho_{r 0}+\frac{\Lambda c^{2}}{8 \pi k}\right)-H_{0}^{2}\right) .
$$

Let us define as usually the density parameters

$$
\Omega_{m}=\frac{8 \pi k \rho_{m 0}}{3 H_{0}^{2}}, \Omega_{r}=\frac{8 \pi k \rho_{r 0}}{3 H_{0}^{2}}, \Omega_{\Lambda}=\frac{\Lambda c^{2}}{3 H_{0}^{2}}
$$

and put

$$
K_{0}=\left(\Omega_{m}+\Omega_{r}+\Omega_{\Lambda}-1\right) / \Omega_{m},
$$

then relation (31) can be rewritten

$$
\frac{8 k c^{4} \lambda}{H_{0}^{2}}-\left(\frac{\varphi_{0}}{H_{0}}\right)^{2}=12 \Omega_{m} K_{0} .
$$


It follows from (27) by the use of (28), (30) and elimination of $h$ and $\dot{h}$ the differential equation

$$
\left(\frac{\dot{a}}{a}\right)^{2}=\frac{H_{0}^{2}}{\left(2 \kappa c^{4} \lambda t^{2}+\varphi_{0} t+1\right)^{2}}\left[-\Omega_{m} K_{0}+\Omega_{r} a^{2}+\Omega_{m} a^{3}+\Omega_{\Lambda} a^{6}\right]
$$

with the initial condition

$$
a(0)=1 \text {. }
$$

A necessary and sufficient condition to avoid singular solutions of (34) is the condition

$$
K_{0}>0 \text {. }
$$

This yields

$$
2 \kappa c^{4} \lambda t^{2}+\varphi_{0} t+1>0
$$

for all $t \in \mathfrak{R}$. Hence, we get a non-singular solution, i.e. we receive a non-singular universe for all $t \in \mathfrak{R}$. It exists $t_{1}<t_{0}=0$ such that

$$
\dot{a}\left(t_{1}\right)=0 \text {. }
$$

Put $a_{1}=a\left(t_{1}\right)$ then it follows from (34a) with $t=t_{1}$

$$
\Omega_{r} a_{1}^{2}+\Omega_{m} a_{1}^{3}+\Omega_{\Lambda} a_{1}^{6}=\Omega_{m} K_{0} .
$$

It follows for all $t \in \mathbb{R}$

$$
a(t) \geq a_{1}>0 .
$$

Subsequently let us assume

$$
a_{1} \ll a(0)=1,
$$

then we get by (38)

$$
K_{0} \ll 1
$$

It follows from (32) by the use of (41)

$$
\Omega_{r}+\Omega_{m}+\Omega_{\Lambda}=1+\Omega_{m} K_{0} .
$$

That is the sum of the density parameters is a little bit greater than 1 . The solution of (34) is by longer calculations and under the assumption $p_{r 0}=0$

$$
\begin{gathered}
a^{3}(t)=2 a_{1}^{3}\left(1+\frac{\Omega_{\Lambda}}{\Omega_{m}} a_{1}^{3}\right) /\left(1+\left(1+2 \frac{\Omega_{\Lambda}}{\Omega_{m}} a_{1}^{3}\right) \cos (\sqrt{3} \beta(t))\right), \\
\beta(t)=\operatorname{arctg}\left(\frac{\sqrt{3 \Omega_{m} K_{0}} H_{0}\left(t-t_{1}\right)}{1+\frac{1}{2} \frac{\varphi_{0}}{H_{0}} t_{1}+(B) H_{0} t}\right),(B)=\left(\left(\left(\frac{1}{2} \frac{\varphi_{0}}{H_{0}}\right)^{2}+3 \Omega_{m} K_{0}\right) H_{0} t_{1}+\frac{1}{2} \frac{\varphi_{0}}{H_{0}}\right) .
\end{gathered}
$$

We get as $t \rightarrow-\infty$

$$
a(-\infty) \approx\left(\frac{2}{(1-\cos (\sqrt{3} \pi))}\right)^{\frac{1}{3}} a_{1} \approx 1.81 a_{1} .
$$


Hence, $a(t)$ starts at $t=-\infty$ with $a(-\infty)=1.81 a_{1}$, decreases to $a_{1}>0$ and then increases for all $t$. It follows from (30) by the use of (43b) for $t$ sufficiently large

$$
\sqrt{h(t)} \approx\left(\frac{1}{2} \frac{\varphi_{0}}{H_{0}} t+1\right)^{2} / a^{3}(-\infty) .
$$

Hence, $a(t)$ starts from a small positive value decreases to a small positive value and then increases for all $t \in \mathcal{R}$. The function $h(t)$ can then be calculated by relation (30).

The proper-time from the beginning of the universe till time $t$ is

$$
\tilde{\tau}(t)=\int_{-\infty}^{t} 1 / \sqrt{h(t)} \mathrm{d} t,
$$

i.e. the age of the universe is finite by GFST analogues to that of GR.

The metric (1) is not expanding, i.e. the universe is not expanding and not singular. In the beginning of the universe it consists only of gravitational energy and no matter and no radiation exist. They arise in the course of time at coasts of gravitational energy and the sum of the total energy is always conserved.

Introducing the proper-time $\tilde{\tau}$ into the differential Equation (34a) we get by the use of (30) the differential equation (see [1])

$$
\left(\frac{1}{a} \frac{\mathrm{d} a}{\mathrm{~d} \tau}\right)^{2}=H_{0}^{2}\left(-\frac{\Omega_{m} K_{0}}{a^{6}}+\frac{\Omega_{r}}{a^{4}}+\frac{\Omega_{m}}{a^{3}}+\Omega_{\Lambda}\right) .
$$

This differential equation is for $a(t)$ sufficiently large (that is: away from the beginning of the universe) by virtue of (41) identical with that of GR. Hence, the function $a(t)$ approximates the scaling factor of GR. In the beginning of the universe the function $a(t)$ is positive whereas the scaling factor of GR is zero what implies by GR the singularity of the expanding universe in the beginning. It is worth to mention that the resulting universes of GR and of GFST are very different. The results of GFST are contained in the book [1] and in article [2]. Additional results about the universe by GFST are found in the articles [3] [4] [5] [6].

A non-expanding universe is experimentally stated by Lerner [7] [8]. The redshift in a non-expanding space is given in [9] and Siegel [10] also asked the question of an expanding space. I must also mention the book of Fahr [11] where a universe without big bang is propagated. In article [12], the redshift of distant objects is derived from the frequency shift of the gravitational field which is changed by converting gravitation to matter.

\section{Redshift of Distant Objects}

It is useful to introduce the absolute time to simplify the calculations of the redshift in the universe. The absolute time $t^{\prime}$ of the universe is given by

$$
\mathrm{d} t^{\prime}=\frac{1}{a(t) \sqrt{h(t)}} \mathrm{d} t=\frac{1}{a(t)} \mathrm{d} \tilde{\tau} .
$$

The proper-time (6) with (21) by the universe is 


$$
(c \mathrm{~d} \tau)^{2}=-a(t)^{2}\left(\sum_{i=1}^{3}\left(\mathrm{~d} x^{i}\right)^{2}-\mathrm{d} c t^{\prime 2}\right) .
$$

The energy of a photon emitted at a distant object at time $t_{e}^{\prime}$ is given by $E\left(t_{e}^{\prime}\right) \sim-g_{44} \frac{\mathrm{d} t^{\prime}}{\mathrm{d} \tilde{\tau}} \rightarrow a\left(t_{e}^{\prime}\right) E_{0}$, which means for the frequencies

$$
v_{e}=a\left(t_{e}^{\prime}\right) v_{0} .
$$

Here, $v_{0}$ is the frequency of the same atom emitted at present. This gives for the frequency moving in the universe by virtue of constant light velocity (see (48))

$$
v(t)=v_{e}=a\left(t_{e}^{\prime}\right) v_{0} .
$$

The redshift is

$$
z=\frac{v_{0}}{v\left(t_{e}^{\prime}\right)}-1=\frac{1}{a\left(t_{e}^{\prime}\right)}-1 .
$$

Light emitted at distance $r$ at time $t_{e}^{\prime}$ and received at $r=0$ at time $t_{0}^{\prime}$ holds by the constant velocity of light

$$
r=c\left(t_{0}^{\prime}-t_{e}^{\prime}\right) \text {. }
$$

This gives by Taylor expansion of $a\left(t_{e}^{\prime}\right)$ and relation (50)

$$
z=H_{0} \frac{r}{c}+\left(1-\frac{1}{2} \frac{1}{H_{0}^{2}} \frac{\mathrm{d}^{2} a\left(t_{e}^{\prime}\right)}{\mathrm{d} t_{e}^{\prime 2}}\right)\left(H_{0} \frac{r}{c}\right)^{2}
$$

The differential Equation (46) is by introducing the absolute time $t^{\prime}$ rewritten

$$
\left(\frac{\mathrm{d} a}{\mathrm{~d} t^{\prime}}\right)^{2}=\frac{H_{0}^{2}}{a^{2}}\left(-\Omega_{m} K_{0}+\Omega_{r} a^{2}+\Omega_{m} a^{3}+\Omega_{\Lambda} a^{6}\right) .
$$

Differentiation of this relation implies by neglecting small expressions

$$
\frac{\mathrm{d}^{2} a}{\mathrm{~d} t_{e}^{\prime 2}}=H_{0}^{2}\left(1-\frac{1}{2} \Omega_{m}+\Omega_{\Lambda}\right) .
$$

Hence, we get by introducing this expression in the relation for $Z$

$$
z \cong H_{0} \frac{r}{c}+\frac{3}{4} \Omega_{m}\left(H_{0} \frac{r}{c}\right)^{2} .
$$

This expression for the redshift is derived by the use of the frequency caused by the change of gravitation and not by a Doppler effect. It was already derived in previous articles (see e.g. the book [1]).

Some additional remarks to GFST:

Gravitational field is attracting. Spherically symmetric perturbations of the gravitational field in the universe attract and are partly converted to matter, an object arises. The rest of this gravitational field surrounds the object and may attract other objects. This seems that matter attracts matter but it is the gravitational field which is attracting. The process of arising objects is fast and implies big objects and may explain the galaxies in our universe. This result can be 
found in [13] and in the book [1]. This gives also an explanation of gravitation. Gravitational field is attracting and not the mass. The densified gravitational field is converted to matter. Matter is the result of the attracting gravitation. This result can be found in article [14].

\section{Conflicts of Interest}

The author declares no conflicts of interest regarding the publication of this paper.

\section{References}

[1] Petry, W. (2014) A Theory of Gravitation in Flat Space-Time. Science PG. https://doi.org/10.4236/jmp.2014.516156

[2] Petry, W. (2015) Journal Modern Physics, 6, 1085-1094. https://doi.org/10.4236/jmp.2015.68113

[3] Petry, W. (2014) Journal of Applied Mathematics and Physics, 2, 50-54. https://doi.org/10.4236/jamp.2014.25007

[4] Petry, W. (2013) Journal Modern Physics, 4, 20-25. https://doi.org/10.4236/jmp.2013.47A1003

[5] Petry, W. (2018) Journal Modern Physics, 9, 1441-1447. https://doi.org/10.4236/jmp.2018.97088

[6] Petry, W. (2017) Journal Applied Mathematics and Physics, 5, 862-872.

[7] Lerner, E.J. (1991) The Big Bang Never Happened. Amazon.de. https://www.amazon.de/dp/0812918533/ref=cm sw $\mathrm{r}$ awdo navT a VWQF7EEN I5T1CBEKQBG3

[8] Lerner, E.J. (2014) Universe Is Not Expanding at All. Science News, May.

[9] Petry, W. (2007) Is the Universe really Expanding? arXiv: 0705.4859

[10] Siegel, E. (2018) Scientists Can't Agree on the Expanding Universe. https://www.forbes.com/sites/startswithabang/2018/12/06/scientists-cant-agree-on-t he-expanding-universe/?sh $=345 f 692 \mathrm{~d} 5 \mathrm{e} 2 \mathrm{c}$

[11] Fahr, H.-J. (1995) Universum ohne Urknall. Spektrum.

[12] Petry, W. (2016) Journal Modern Physics, 7, 1492-1499. https://doi.org/10.4236/jmp.2016.712135

[13] Petry, W. (1996) Astrophysics and Space-Science, 235, 41-57. https://doi.org/10.1007/BF00643589

[14] Petry, W. (2019) Journal Modern Physics, 10, 157-162. https://doi.org/10.4236/jmp.2019.102012 